\title{
Alterações da pressão arterial e glicemia capilar entre trabalhadores da educação
}

\author{
Alterations on blood pressure and glycemia between education \\ Cambios en la presión arterial y la glucemia capilar entre trabajadores de la educación
}

\section{Resumo}

Objetivo: descrever os casos de alteração de pressão arterial e de glicemia capilar entre trabalhadores da educação de colégio universitário. Metodologia: estudo epidemiológico observacional, descritivo seccional. A coleta de dados ocorreu durante o ano de 2018. A amostra de estudo foi composta de trabalhadores de colégio universitário. O total de participantes foi de 106 profissionais. Resultados: entre os funcionários, 47 (44,9\%) apresentaram pressão alterada, e $12(11,3 \%)$ a glicemia elevada. Na análise bivariada, entre pressão arterial e aspectos sociodemográficos, laborais e de saúde observou-se associação, entre o sexo masculino $(\mathrm{p}=0,007)$, idade acima da média de 38 anos $(\mathrm{p}=0,005)$, tempo no setor acima de 4 anos $(\mathrm{p}=0,004)$, perímetro abdominal elevado $(\mathrm{p}=0,009)$ e sobrepeso e obesidade $(\mathrm{p}=0,001)$. Não houve significância estatística entre a glicemia capilar e as variáveis investigadas. Conclusão: destacam-se o sexo masculino, a idade, perímetro abdominal e o sobrepeso como características que se relacionam com os níveis pressóricos elevados. As medidas de promoção à saúde e qualidade de vida devem ser estimuladas entre o grupo de trabalhadores.

Palavras-chave: Enfermagem do trabalho; Doenças não transmissíveis; Saúde do trabalhador.

\begin{abstract}
Objective: to describe the cases of changes in blood pressure and capillary glycemia among university education workers. Methodology: observational, sectional descriptive epidemiological study. Data collection took place during 2018. The study sample consisted of university college workers. The total number of participants was 106 professionals. Results: among the employees, 47 (44.9\%) had altered blood pressure, and 12 (11.3\%) had high blood glucose. In the bivariate analysis, between blood pressure and sociodemographic, occupational and health aspects, an association was observed between males $(\mathrm{p}=0.007)$, age above the mean of 38 years $(\mathrm{p}=0.005)$, time in the sector above 4 years $(p=0.004)$, high abdominal circumference $(p=0.009)$ and overweight and obesity $(p=0.001)$. There was no statistical significance between capillary blood glucose and the variables investigated. Conclusion: male gender, age, abdominal circumference and overweight stand out as characteristics that are related to high blood pressure levels. Measures to promote health and quality of life should be encouraged among the group of workers.
\end{abstract} Keywords: Occupational nursing; Noncommunicable diseases; Worker's health. 


\begin{abstract}
Resumen
Objetivo: describir los casos de alteraciones de la presión arterial y de la glucemia capilar en trabajadores de la educación universitaria. Metodología: estudio epidemiológico descriptivo, observacional, seccional. La recolección de datos se llevó a cabo durante 2018. La muestra del estudio estuvo compuesta por trabajadores de colegios universitarios. El total de participantes fue de 106 profesionales. Resultados: entre los empleados, 47 (44,9\%) tenían tensión arterial alterada y $12(11,3 \%)$ tenían glucemia elevada. En el análisis bivariado, entre presión arterial y aspectos sociodemográficos, ocupacionales y de salud, se observó asociación entre hombres $(\mathrm{p}=0,007)$, edad superior a la media de 38 años $(\mathrm{p}=0,005)$, tiempo en el sector superior a 4 años $(\mathrm{p}=0,004)$, circunferencia abdominal elevada $(\mathrm{p}=0,009)$ y sobrepeso y obesidad $(\mathrm{p}=0,001)$. No hubo significación estadística entre la glucemia capilar y las variables investigadas. Conclusión: el sexo masculino, la edad, la circunferencia abdominal y el sobrepeso se destacan como características que se relacionan con la hipertensión arterial. Deberían fomentarse medidas para promover la salud y la calidad de vida entre el grupo de trabajadores.

Palabras clave: Enfermería del trabajo; Enfermedades no transmisibles; Salud del trabajador.
\end{abstract}

\title{
1. Introdução
}

A rapidez dos processos de globalização e urbanização fez com que os brasileiros, assim como a população mundial, adotassem um estilo de vida sedentário, aliado ao consumo de alimentos industrializados, além de alimentos com excesso de calorias, lipídios, sal, açúcares e conservantes. Somando-se a isso, o uso frequente de tabaco e consumo de álcool favorecem o aparecimento de doenças crônicas não transmissíveis (DCNT) na população (Morais et al., 2018).

As DCNT, como as doenças cardiovasculares e respiratórias crônicas, diabetes mellitus (DM) e neoplasias, são condições muito prevalentes e de origem multifatorial, resultante de determinantes biológicos e socioculturais (Ministério da Saúde, 2014). Estimativas apontam que tais doenças constituem a principal causa de morte no mundo, representando $71 \%$ das 57 milhões de mortes ocorridas no mundo em 2016, sendo igualmente relevantes no Brasil, tendo causado $74 \%$ do total de mortes em 2016, com destaque para doenças cardiovasculares (28\%) e DM (5\%) (Vigitel 2019).

Atualmente, o Brasil passa por mudanças epidemiológicas no padrão de saúde-doença. Para Bahia e Araújo (2014), o aumento considerável de doenças crônicas, dentre estas DM e hipertensão arterial (HAS), correspondentes pela maior causa de mortalidade e internações hospitalares, somado à epidemia global de sobrepeso e à obesidade contribuem para incidência das DCNT e aumento dos custos em saúde.

A hipertensão arterial (HA) é uma condição clínica multifatorial caracterizada por níveis elevados e sustentados de pressão arterial - PA (PA $\geq 140 / 90 \mathrm{mmHg}$ ) (Ministério da Saúde, 2013). Por sua vez, o DM é uma doença metabólica, caracterizada por hiperglicemia e distúrbios no metabolismo de carboidratos, proteínas e gorduras, resultantes de problemas na secreção e/ou da ação da insulina (Ministério da Saúde, 2013).

Sabe-se que a hipertensão arterial e o diabetes mellitus, quando não controladas, podem acarretar em complicações para o indivíduo. O DM está habitualmente associado à dislipidemia, a hipertensão arterial sistêmica e à disfunção endotelial. Frequentemente, a HAS está associada a distúrbios metabólicos e alterações em órgãos-alvo, sendo agravada pela presença de outros fatores de risco, como dislipidemia, obesidade abdominal, intolerância à glicose e DM (Malachias et al., 2016).

Devido à relevância das DCNT na influência do perfil epidemiológico da população brasileira, somado ao fato de que a maior parte de seus determinantes está sujeita à prevenção, nota-se a importância de ações e políticas públicas voltadas para o enfrentamento destas comorbidades (Vigitel, 2019).

A enfermagem possui papel fundamental nesse processo de enfrentamento, através da realização de consultas, da abordagem dos fatores de risco, do esclarecimento sobre as terapias não farmacológicas e na adesão ao tratamento. Além disso, destaca-se a sua atuação na promoção da saúde, educando individualmente ou em grupo, os pacientes (Ministério da Saúde, 2013).

Objetivando atuar na promoção de saúde e prevenção de doenças crônicas, como a HAS e DM, com profissionais da educação deve-se entender que o trabalho é parte integrante e fundamental para a vida. Sendo o trabalho docente constituído 
por muitas tarefas extras-classe, múltiplas jornadas de trabalho, prazos curtos e demandas excessivas, expondo essa categoria a ambientes estressantes, impactando diretamente na saúde desses indivíduos (Silva, Andrade, Pereira, \& Silva, 2010).

O exercício do trabalho se faz presente em pelo menos um terço do dia do trabalhador, o que faz com que exerça enorme influência sobre sua vida, pois o trabalho interliga o trabalhador com o meio em que vive, interferindo em sua saúde e nas dimensões da política, da sociedade, da cultura e de sua família (Guerreiro, Nunes, Gonzalez, \& Mesas, 2016; Vasconcelos, 2011).

A condição de saúde é um aspecto fundamental para a qualidade de vida bem como para a capacidade de trabalho das pessoas (Santos \& Marques, 2013). No campo da educação, tem sido evidenciado um crescimento quanto ao número de agravos relacionados à saúde dos trabalhadores, sendo frequentemente associados às características e condições de trabalho existentes (Cruz \& Lemos, 2005; Gasparini, Barreto, \& Assunção, 2005).

A ocorrência de doenças crônicas relacionadas ao estilo de vida tem adquirido relevância na categoria docente, especialmente devido aos hábitos de vida que incluem a inatividade física, alimentação inadequada e consumo de álcool ou tabaco (Santos \& Marques, 2013).

Ao compreender como as DCNT podem afetar a qualidade de vida dos trabalhadores da educação, o presente estudo traz como problema de pesquisa: Existem casos de alteração de pressão arterial e glicemia capilar entre os profissionais da educação?

O objetivo dessa pesquisa é descrever os casos de alteração de pressão arterial e de glicemia capilar entre profissionais da educação de um colégio universitário na cidade de Niterói-RJ, investigando fatores associados à sua ocorrência.

\section{Metodologia}

Estudo epidemiológico observacional, descritivo seccional. O estudo seguiu a resolução 466/2012(*), e contou com a aprovação do Comitê de Ética do Hospital Universitário Antônio Pedro, sob o número 2.324.524.

Os estudos seccionais possuem característica descritiva, entretanto em virtude da busca da associação estatística aplicando de modelos de regressão logística, entende-se que o aprofundamento conduzirá a uma análise mais apurada entre e exposição e desfecho. A tipologia permite o estudo de múltiplos fatores ao mesmo tempo (Klein; Bloch, 2006, p.127).

Estudos seccionais têm sido utilizados para investigar uma gama de problemas em saúde pública. De modo geral, a aplicação mais comum do estudo deste tipo está ligada à necessidade de conhecer de que maneira se distribuem uma ou mais características individuais ou coletivas em determinada população (Klein; Bloch, 2006, p.127).

A coleta de dados ocorreu durante o ano de 2018. A amostra de estudo foi composta de trabalhadores (docentes, pedagogos, administrativos, segurança, apoio, cozinha e demais funcionários) de colégio universitário na cidade de Niterói RJ. O total de participantes foi de 106 profissionais.

Os critérios de inclusão foram: indivíduos adultos entre 18 e 60 anos, funcionários do colégio, de ambos os sexos, independente de cor de pele e religião. Foram excluídos os aposentados, maiores de 60 anos, grávidas e afastados do trabalho por mais de seis meses.

O instrumento utilizado para a coleta de dados foi um questionário estruturado com perguntas abertas e fechadas, elaborado pelos próprios pesquisadores e que foi utilizado como formulário. O instrumento de coleta de dados contém seções organizadas por assunto, sendo: aspectos relacionados às características sociodemográficas e renda per capita por salário mínimo. Os estratos da variável cor da pele autorreferida baseiam-se em classificação proposta pelo Instituto Brasileiro de Geografia e Estatística (IBGE, 2019): branca, negra, parda, amarela ou indígena. Em relação ao aspecto laboral, foram estudadas as variáveis: categoria profissional, tipo de vínculo empregatício, horário de trabalho, carga horária semanal, número de empregos, setor de trabalho e tempo de atuação no setor. 
Após o preenchimento do formulário, realizou-se a mensuração da pressão arterial e da glicemia capilar, peso, altura, índice cintura-quadril, perímetro abdominal e porcentagem de gordura sob critérios estabelecidos em diretrizes brasileiras (Malachias et al., 2016; Ministério da Saúde, 2014; Sociedade Brasileira de Diabetes, 2018). Deve-se salientar que todas as normas de biossegurança foram seguidas para coleta de dados clínicos, inclusive o descarte e higienização adequadas do material utilizado.

A variável pressão arterial foi analisada por meio da $7^{\text {a }}$ diretriz brasileira de hipertensão arterial, que considera como normotensão uma PA sistólica (PAS) $\leq 120 \mathrm{mmHg}$ e uma PA diastólica (PAD) $\leq 80 \mathrm{mmHg}$, como pré-hipertensão uma PAS entre 121-139 mmHg e/ou PAD entre 81-89 mmHg, como hipertensão grau I uma PAS entre 140-149 mmHG e/ou uma PAD entre 90-99 mmHg e como hipertensão grau II uma PAS $\geq 160$ mmHg e/ou PAD $\geq 100$ mmHg (Malachias et al., 2016).

A medida do perímetro abdominal foi analisada através de método definido pela OMS (2000), em que a aferição é feita com uma fita métrica ao redor do abdômen, na altura do ponto médio entre o rebordo costal inferior e a crista ilíaca, considerando como risco cardiovascular um valor $\geq 94 \mathrm{~cm}$ para homens e $\geq 80 \mathrm{~cm}$ para mulheres, e como alto risco cardiovascular um valor $\geq 102 \mathrm{~cm}$ para homens e $\geq 88 \mathrm{~cm}$ para mulheres (Ministério da Saúde, 2014).

O IMC foi calculado por meio da seguinte fórmula (OMS, 1995) IMC $=\frac{P P_{s o}(\mathrm{kgg})}{\mathrm{Aitura}^{2}(\mathrm{~m})}$ sendo classificado em eutrofia um valor entre $18,5-24,99 \mathrm{~kg} / \mathrm{m}^{2}$, sobrepeso um valor entre $25-29,99 \mathrm{~kg} / \mathrm{m}^{2}$ e obesidade um valor acima de $30 \mathrm{~kg} / \mathrm{m}^{2}$ (Ministério da Saúde, 2014).

Por sua vez, os valores glicêmicos obtidos por meio do teste de glicemia capilar considerados neste estudo obedecem aos critérios estabelecidos pela Sociedade Brasileira de Diabetes (2017-2018), sendo: glicemia de jejum ou pré-prandial 65 a $100 \mathrm{mg} / \mathrm{dL}$; pós-prandial de 80 a $12690 \mathrm{mg} / \mathrm{dL}$; ao deitar 80 a $100 \mathrm{mg} / \mathrm{dL}$ e na madrugada 65 a $100 \mathrm{mg} / \mathrm{dL}$.

$\mathrm{O}$ teste qui-quadrado (X2) foi utilizado para verificar diferenças entre os grupos analisados, durante a análise bivariada. Foi considerado, na avaliação da significância, o valor $\mathrm{p} \leq 0,05$ (Hosmer \& Lemeshow, 1989).

$\mathrm{Na}$ análise dos dados, buscou-se relacionar os achados com o referencial teórico abordado aos achados. Cada etapa do processo de análise dos dados foi realizada utilizando o programa Statistical Package for the Social Sciences versão 21 (SPSS $®$ ).

\section{Resultados}

Participaram da pesquisa 106 trabalhadores do colégio universitário. Entre os trabalhadores, 64 se declararam brancos (60,4\%); em relação ao sexo, 79 eram mulheres (74,5\%); a média de idade encontrada foi de 38 anos (DP $\pm 12,4)$, com 47 acima (44,3\%); no quesito escolaridade, 57 cursaram até o ensino superior completo (53,8\%); quanto ao estado civil, 54 viviam sem companheiro (a) (50,9\%); 54 não possuíam filhos (as) (50,9\%); a renda média per capita foi entre 4 e 5 salários mínimos.

Quanto à categoria profissional, $54(50,9 \%)$ professores e $52(49,1 \%)$ apoio; a maioria possuía um vínculo empregatício $(80,2 \%)$, faziam parte do quadro permanente da instituição (58,5\%), trabalhavam em turno integral $(84,9 \%)$, a média de tempo no setor aferida foi de 4 anos (DP $\pm 5,7)$, com 74 (69,8\%) profissionais abaixo dessa média; quanto ao tempo na instituição, a média foi de 5 anos (DP $\pm 7,8)$, com 80 (75,5\%) funcionários com tempo inferior a essa faixa; a carga horária semanal média encontrada foi de $33 \mathrm{~h}$ (DP $\pm 0,5 \%)$, com $63(59,4 \%)$ acima desse valor.

Sobre os hábitos de vida, 98 (92,5\%) dos funcionários relataram não fumar; quanto ao consumo de bebida alcoólica $62(58,5 \%)$ declararam não consumir; $54(50,9 \%)$ dos trabalhadores não praticam exercício físico. Quanto à alimentação 56 $(52,8 \%)$ consomem uma a três vezes na semana frituras, $38(35,8 \%)$ consomem uma a três vezes na semana produtos industrializados, $54(50,9 \%)$ consomem diariamente frutas e $60(56,6 \%)$ consomem verduras diariamente.

Entre os funcionários, 47 (44,9\%) apresentaram pressão alterada considerando os estratos de risco (Tabela 1). A 
média da cifra sistólica foi de 112, e a diastólica $72 \mathrm{mmHg}$. O desvio padrão foi de 13 e $14 \mathrm{mmHg}$, respectivamente. A maior pressão sistólica encontrada foi de $160 \mathrm{mmHg}$, e a diastólica de $130 \mathrm{mmHg}$.

Tabela 1. Distribuição dos valores pressóricos, segundo parâmetros da $7^{\mathrm{a}}$ diretriz brasileira de hipertensão arterial, de trabalhadores de colégio universitário, Niterói (2018).

\begin{tabular}{llc}
\hline Estratos da pressão arterial & N & $\%$ \\
\hline Normotenso (PA < 120/80 mmHg) & 59 & 55,1 \\
Pré-hipertenso (PA entre 120/80 e 139/89mmHg) & 35 & 33,0 \\
Hipertensão grau I (PA entre 140/90 e 159/99mmHg) & 10 & 9,4 \\
Hipertensão grau II (PA igual ou >160/100mmHg) & 02 & 2,5 \\
\hline
\end{tabular}

Legenda: $\mathrm{N}=$ frequência simples, \% frequência relativa. Fonte: Elaboração própria.

A glicemia elevada, seja pós-prandial ou não, foi encontrada entre 12 indivíduos (11,3\%), a média foi de 116 mg, com desvio padrão de $27 \mathrm{mg} /$ decilitros. O valor mínimo encontrado foi de 78 e o máximo de $261 \mathrm{mg} /$ decilitros, a distribuição pode ser observada, na Figura 1.

Figura 1. Distribuição dos valores glicêmicos de trabalhadores de colégio universitário, Niterói (2018).

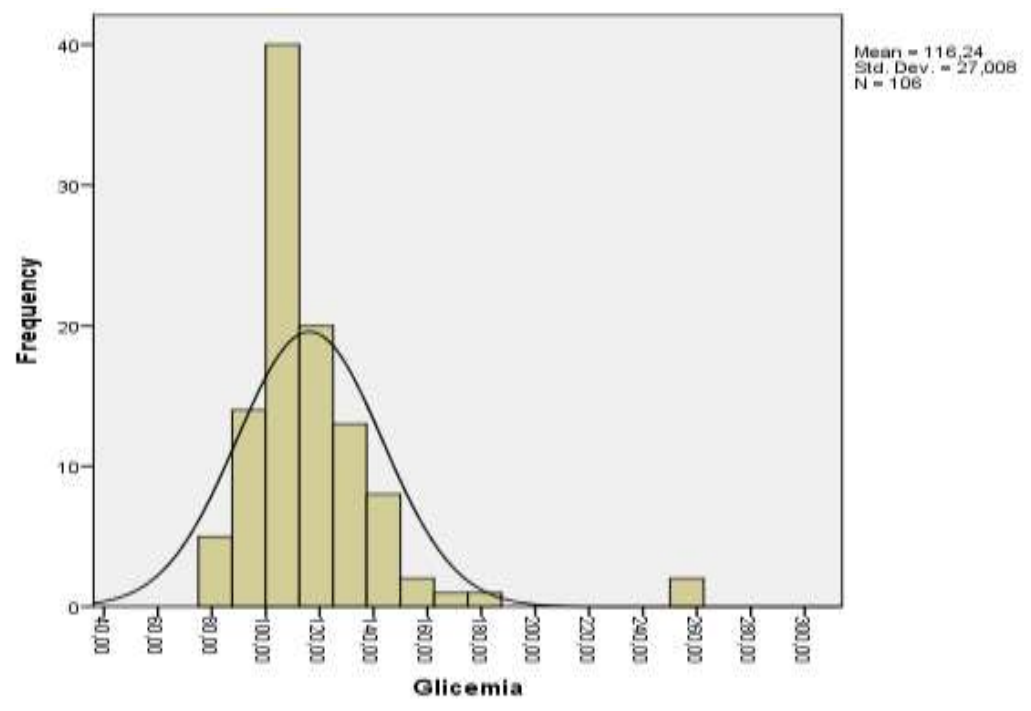

Fonte: Elaboração própria.

Ainda sobre dados clínicos, 84 (79,2\%) apresentaram percentual de gordura corporal elevado, e de acordo com o perímetro abdominal, 42 (39,6\%) apresentaram alto risco, 27 (25,5\%) risco, enquanto que somente 37 (34,9\%) apresentaram perímetro ideal. De acordo com a classificação de índice de massa corporal (IMC), 66 (62,3\%) trabalhadores apresentam sobrepeso/obesidade(6,7).

A Tabela 2, a seguir, demonstra a análise bivariada realizada para calcular o nível de significância estatística entre as variáveis sociodemográficas, laborais e de saúde com a pressão arterial. As variáveis: idade; sexo; filhos; escolaridade; tipo de vínculo; tempo no setor; perímetro abdominal e IMC apresentaram significância estatística ( $p<=0,05$ ). 
Tabela 2. Alteração da pressão arterial, de acordo com variáveis sociodemográficas, laborais e de saúde entre trabalhadores de colégio universitário, Niterói (2018).

\begin{tabular}{|c|c|c|c|c|}
\hline VARIÁVEIS & $\mathbf{N}$ & $\mathbf{N}$ & $\%$ & Valor de $p$ \\
\hline Idade & & & & 0,005 \\
\hline Até 38 anos & 59 & 19 & 32,20 & \\
\hline Acima de 38 anos & 47 & 28 & 59,57 & \\
\hline Sexo & & & & $\mathbf{0 , 0 0 7}$ \\
\hline Feminino & 79 & 29 & 36,70 & \\
\hline Masculino & 27 & 18 & 66,67 & \\
\hline Filhos & & & & $\mathbf{0 , 0 5 0}$ \\
\hline Não & 15 & 11 & 73,33 & \\
\hline Escolaridade & & & & 0,015 \\
\hline Até o ensino superior incompleto & 20 & 04 & 20,00 & \\
\hline Acima do ensino superior incompleto & 86 & 43 & 50,00 & \\
\hline Tipo de vínculo & & & & $\mathbf{0 , 0 1 0}$ \\
\hline Temporário & 44 & 13 & 29,54 & \\
\hline Permanente & 62 & 34 & 54,83 & \\
\hline Tempo no setor & & & & 0,004 \\
\hline Até 4 anos & 74 & 26 & 35,13 & \\
\hline Acima de 4 anos & 32 & 21 & 65,62 & \\
\hline Perímetro abdominal & & & & 0,009 \\
\hline Ideal & 37 & 09 & 24,32 & \\
\hline Risco & 27 & 14 & 51,85 & \\
\hline Alto risco & 42 & 24 & 57,14 & \\
\hline IMC & & & & $0,001 *$ \\
\hline Ideal & 40 & 09 & 22,50 & \\
\hline Sobrepeso/Obesidade & 66 & 38 & 57,57 & \\
\hline
\end{tabular}

Legenda: N (população total), n (suspeitos), \% (prevalência), *(Teste de Fisher). Fonte: Elaboração própria.

A Tabela 3, a seguir, demonstra a análise bivariada realizada para calcular o nível de significância estatística entre as variáveis sociodemográficas, laborais e de saúde com a glicemia capilar. 
Tabela 3. Alterações glicêmicas, de acordo com variáveis sociodemográficas, laborais e de saúde entre trabalhadores de colégio universitário, Niterói (2018).

\begin{tabular}{|c|c|c|c|c|}
\hline VARIÁVEIS & $\mathbf{N}$ & $\mathbf{N}$ & $\%$ & Valor de $\mathbf{p}^{*}$ \\
\hline Idade & & & & 0,300 \\
\hline Até 38 anos & 59 & 05 & 08,47 & \\
\hline Acima de 38 anos & 47 & 07 & 14,89 & \\
\hline Sexo & & & & 0,507 \\
\hline Feminino & 79 & 08 & 10,12 & \\
\hline Masculino & 27 & 04 & 14,81 & \\
\hline Filhos & & & & 0,470 \\
\hline Não & 15 & 03 & 20,00 & \\
\hline Sim & 37 & 03 & 08,10 & \\
\hline Escolaridade & & & & 0,076 \\
\hline Até o ensino superior incompleto & 20 & 00 & 00,00 & \\
\hline Acima do ensino superior incompleto & 86 & 12 & 13,95 & \\
\hline Tipo de vínculo & & & & 0,612 \\
\hline Temporário & 44 & 05 & 11,36 & \\
\hline Permanente & 62 & 07 & 11,29 & \\
\hline Tempo no setor & & & & 0,112 \\
\hline Até 4 anos & 74 & 06 & 08,10 & \\
\hline Acima de 4 anos & 32 & 06 & 18,75 & \\
\hline Perímetro abdominal & & & & 0,674 \\
\hline Ideal & 37 & 04 & 10,81 & \\
\hline Risco & 27 & 02 & 07,40 & \\
\hline Alto risco & 42 & 06 & 14,28 & \\
\hline IMC & & & & 0,765 \\
\hline Ideal & 40 & 05 & 12,50 & \\
\hline Sobrepeso/Obesidade & 66 & 07 & 10,60 & \\
\hline
\end{tabular}

Legenda: N (população total), n (suspeitos), \% (prevalência), *(Teste de Fisher). Fonte: Elaboração própria.

Em relação à análise realizada com as variáveis investigadas com a glicemia capilar, notou-se que nenhuma apresentou significância estatística.

\section{Discussão}

Nesta pesquisa, a quantidade de mulheres que participaram do estudo foi maior do que a de homens $(74,52 \%)$. A predominância feminina deve-se aos fatores socioculturais que levaram as mulheres a ocupar majoritariamente as vagas de trabalho no campo da educação (Santos \& Marques, 2013). Estudos comprovam frequentes alterações na pressão arterial em 
docentes (Oliveira, Júnior, Tavares, Moreira, \& Marins, 2015; Correia, Lima, Lucena, \& Silva, 2017). No presente estudo, os homens foram os que apresentaram significância estatística com a pressão arterial alterada na análise bivariada $(\mathrm{p}=0,007)$.

Tal achado assemelha-se ao estudo transversal realizado com 150 docentes (Santos et al., 2017), onde os homens foram os que apresentaram valores mais alterados de PA $(\mathrm{p}=0,0001)$. Estudo de Moreira et al (2011) realizado com professores também apontou significância estatística entre homens e valores alterados de $P A(p<0,05)$. Entretanto, normalmente, pesquisas comprovam que o sexo feminino tem maior prevalência de diagnósticos de HAS (Barreto, Mendonça, Pimenta, Garcia-Vivar, \& Marcon, 2018; Pereira et al., 2018).

Em relação à idade, a média encontrada nos sujeitos da pesquisa foi de 38 anos. A variável idade também apresentou significância estatística quando avaliada com a pressão arterial alterada $(\mathrm{p}=0,005)$. Pesquisas também encontraram associação positiva entre essas variáveis $(\mathrm{p}=0,048)$, relacionando o aumento de idade na maior possibilidade de haver alteração na PA. Outros estudos também relatam tal associação (Santos et al., 2017; Moreira et al., 2011; Barreto et al., 2018; Pereira et al.,2018). Sabe-se que dentre os fatores de risco não modificáveis para a ocorrência de HAS se encontram o sexo e a idade (Santos et al., 2017). A variável escolaridade também apresentou significância estatística ( $\mathrm{p}=0,015)$, quando analisada com a PA. Neste estudo, os sujeitos da pesquisa que apresentaram maior índice de escolaridade (acima de superior incompleto), possuem maior probabilidade de apresentarem níveis pressóricos elevados, tal fator pode ter relação com poder aquisitivo e estilo de vida que favoreça hábitos de saúde maléficos. Nota-se que esse aspecto destoa de outros estudos, que atestam que os indivíduos com baixa escolaridade possuem maior probabilidade de apresentarem PA elevada (Pereira et al., 2018; Carvalho et al., 2016; Silva et al., 2016).

Em relação às variáveis laborais, o vínculo permanente e o tempo no setor acima de quatro anos apresentaram significância estatística, ( $p=0,010$ e 0,004, respectivamente), o que demonstra que a estabilidade no trabalho pode estar relacionada ao objeto deste estudo.

Notou-se, ainda, que os valores de IMC e a circunferência abdominal tiveram associação significativa com a pressão arterial elevada ( $p=0,009$ e 0,001, respectivamente), o que corrobora com os achados de outros estudos, como um estudo transversal realizado com 414 professores municipais de Bagé (RS) que constatou excesso de peso (sobrepeso e obesos) entre os profissionais, além da prevalência de 20,3\% de hipertensão (Santos \& Marques, 2013). Outros autores também encontraram associação significativa entre o IMC alterado e índices elevados de PA, o que corrobora os achados deste artigo (Santos et al., 2017).

Saraiva et al (2011) Ao analisar o sobrepeso e a obesidade entre 67 docentes de instituição privada de ensino de Fortaleza, constatou que a obesidade foi identificada em 33\% da amostra e o sobrepeso em 14,5\% dos professores. O excesso de peso estava mais presente em homens $(p<0,05)$ e nos indivíduos com faixa etária entre 36 e 45 anos. Além disso, foi encontrado um percentual elevado (60\%) de excesso de peso entre docentes de universidade pública de Minas Gerais (Xavier, Barboza, Monteiro, Santos, \& Oliveira, 2010). Essas expressivas prevalências de fatores de risco cardiovascular, como o excesso de peso, denotam a importância da realização de ações de promoção da saúde para esta população. O grupo de pesquisadores devolveu os achados aos participantes, por meio de palestras, em reuniões de docentes e panfletos educativos sobre os temas, além dos estudos científicos divulgados.

Algumas limitações devem ser consideradas nesse estudo, entre elas destaca-se a dinâmica de trabalho, pois a coleta de dados se deu no horário de trabalho dos funcionários, que eram abordados, antes ou após o turno de trabalho, ou durante o expediente, no horário de intervalo, pois vale ressaltar, que a pressão arterial sofre alterações fisiológicas durante o dia, o que pode ocasionar pequenas alterações dos níveis pressóricos no momento da mensuração de tais valores (Silva, 2017).

Sobre o viés do trabalhador saudável, alguns funcionários estavam de licença médica, durante o período da coleta; entretanto, após seu retorno, foram inseridos como participantes do estudo. Além disso, foram solicitadas ao setor médico 
ocupacional a relação de trabalhadores em licença médica, e estes ao retornarem foram convidados a aceitaram participar, essas iniciativas minimizaram o possível problema.

\section{Conclusão}

A partir deste estudo foi possível identificar que o sexo masculino, idade acima de 38 anos, escolaridade acima do nível superior incompleto, vínculo permanente na instituição, tempo de setor, valor do IMC e da circunferência abdominal foram fatores de risco associados à hipertensão entre trabalhadores da educação.

A associação entre fatores relacionados ao estilo de vida e a presença de níveis alterados de pressão arterial fundamenta a recomendação da literatura quanto à mudança de estilo de vida, objetivando a adoção de melhores hábitos, que minimizem os fatores de risco para o desenvolvimento da hipertensão arterial, diabetes e outras DCNT.

Além disso, espera-se que os resultados encontrados neste estudo estimulem novas pesquisas sobre as DCNT entre os profissionais da educação e outras categorias, visto que se trata de temática relevante. A saúde se constrói com conhecimento e prevenção, evitando a instauração de doenças.

Para estudos futuros, sugerimos que os dados possam ser coletados em um menor espaço de tempo, com menos interferências da dinâmica de trabalho desses profissionais, além de utilizar uma amostra maior, possibilitando maiores comparações com as obras disponíveis na literatura atual. Dessa forma, poderemos conhecer, prevenir e tratar cada vez mais tais patologias responsáveis por grande parte da morbimortalidade da população brasileira e mundial.

\section{Referências}

Barreto, M. S., Mendonça, R. D., Pimenta, A. M., Garcia-Vivar, C. \& Marcon, S. S. (2018). Não utilização de consultas de rotina na Atenção Básica por pessoas com hipertensão arterial. Ciência \& Saúde Coletiva, 23(3), 795-804. http://dx.doi.org/10.1590/1413-81232018233.12132016.

Bahia, L. R. \& Araújo, D. V. (2014). Impacto econômico da obesidade no Brasil. Rev HUPE, 13 (1): 13-7. https://www.epublicacoes.uerj.br/index.php/revistahupe/article/view/9793.

Brasil. Ministério da Saúde. Vigitel Brasil. (2018). Vigilância de fatores de risco e proteção para doenças crônicas por inquérito telefônico. https://bvsms.saude.gov.br/bvs/publicacoes/vigitel_brasil_2017_vigilancia_fatores_riscos.pdf.

Brasil. Ministério da Saúde. (2013). Estratégias para o cuidado da pessoa com doença crônica: hipertensão arterial sistêmica. (Cadernos de Atenção Básica, n. 37). http://bvsms.saude.gov.br/bvs/publicacoes/estrategias_cuidado_pessoa_doenca_cronica.pdf.

Carvalho, C. J., Marins, J. C. B., Amorim, P. R. S., Fernandes, M. F., Reis, H. H. T., Sales, S. S., Miranda, M. R. \& Lima, L. M. (2016). Altas taxas de sedentarismo e fatores de risco cardiovascular em pacientes com hipertensão arterial resistente. Medicina, 49(2), 124-33. https://doi.org/10.11606/issn.21767262.v49i2p124-133.

Correia, A. A., Lima, M., Lucena, A. L. R. \& Silva, C. C. (2017). Identificando fatores de risco para a hipertensão arterial sistêmica em professores da rede pública. Rev Enferm UFPE, 11: 264-271. http://dx.doi.org/10.5205/reuol.7995-69931-4-SM.1101sup201703.

Cruz, R. M. \& Lemos, J. C. (2005). Atividade docente, condições de trabalho e processos de saúde. Motrivivência, 24(17):59-80.

Diretrizes da Sociedade Brasileira de Diabetes. (2017-2018). Editora Clannad. https://www.diabetes.org.br/profissionais/images/2017/diretrizes/diretrizessbd-2017-2018.pdf.

Gasparini, S. M., Barreto, S. M \& Assunção, A. A. (2005). O professor, as condições de trabalho e os efeitos sobre sua saúde. Educação e Pesquisa, 31(2):180-199.

Guerreiro, N. P., Nunes, E. F. P. A., Gonzalez, A. D. \& Mesas, A. E. (2016). Perfil sociodemográfico, condições e cargas de trabalho de professores da rede estadual de ensino de um município da região do sul do Brasil. Trab. Ed. Saúde, 14(1), 197-217. https://doi.org/10.25247/P1982-999X.2011.v1n2.p\%25p.

Hosmer, D. W. \& Lemeshow, S. (1989). Applied Logistic Regression. John Wiley \& Sons.

Malachias, M. V. B., Souza, W. K. S. B., Plavnik, F. L., Rodrigues, C. I. S., Brandão, A. A., Neves, M. F. T. et al. (2016). $7^{\text {a }}$ Diretriz Brasileira de Hipertensão Arterial. Arq Bras Cardiol, 107 (3): 1-83. http://publicacoes.cardiol.br/2014/diretrizes/2016/05_HIPERTENSAO_ARTERIAL.pdf.

IBGE. (2019). Cor ou raça. https://educa.ibge.gov.br/jovens/conheca-o-brasil/populacao/18319-cor-ou-raca.html

Klein, C. H. \& Bloch, K. V. (2006). Estudos seccionais. In Epidemiologia (cap. 9). Editora Atheneu. 
Research, Society and Development, v. 10, n. 4, e39610413946, 2021

(CC BY 4.0) | ISSN 2525-3409 | DOI: http://dx.doi.org/10.33448/rsd-v10i4.13946

Ministério da Saúde. Cadernos de Atenção Básica (2014). Estratégias para o cuidado da pessoa com doença crônica. https://bvsms.saude.gov.br/bvs/publicacoes/estrategias_cuidado_pessoa_doenca_cronica_cab35.pdf

Morais, H. C. C., Cavalcante, S. N., Nascimento, L. B., Mendes, I. C., Nascimento, K. P. \& Fonseca, R. (2018). Fatores de risco modificáveis para doenças crônicas não transmissíveis entre estudantes universitários. Rev Rene. 19:e3487. https://doi.org/10.15253/2175-6783.2018193487.

Moreira, O.C., Oliveira, R. A. R., Andrade Neto, F., Amorim, W., Oliveira, C. E. P., Doimo, L. A., Amorim, P. R. S., Laterza, M. C., Monteiro, W. D. \& Marins, J. C. B. (2011). Associação entre risco cardiovascular e hipertensão arterial em professores universitários. Revista Brasileira de Educação Física e Esporte, 25(3), 397-406. http://dx.doi.org/10.1590/S1807-55092011000300005.

Oliveira, R. A. R., Júnior, R. J. M., Tavares, D. D. F., Moreira, O. C. \& Marins, J. C. B. (2015). Fatores associados à pressão arterial elevada em professores da educação básica. Rev Educ Fís, 1: 119-129. http://dx.doi.org/10.4025/reveducfis.v26i1.24693.

Pereira, N. D., Rodrigues, T. F. C. S., Sanches, R. C. N., Scardoelli, M. G. C., Trevisan, F. G., Rêgo, A. S. \& Radovanovic, C. A. T. (2018). Sociodemographic and clinical profile of hypertensive patients accompanied by the Family Health Strategy. O Mundo da Saúde, 42(4), 974-991. http://dx.doi.org/10.15343/0104-7809.20184204974991.

Santos, A. G. Fernandes, E. C. R., Barbosa, E. M. P., Figueiredo, K. A. \& Andrade D. B. (2017). Arterial hypertension and associated risk factors in teachers. Rev Enferm UFPI, 6 (3): 37-44. https://doi.org/10.26694/reufpi.v6i3.5880.

Santos, M. N. \& Marques, A. C. (2013). Condições de saúde, estilo de vida e características de trabalho de professores de uma cidade do sul do Brasil. Ciên Saúde Coletiva, 18 (3): 837-846. http://dx.doi.org/10.1590/S1413-81232013000300029.

Saraiva, F. V., Freitas, R. W. J. F., Sousa, V. E. C., Araújo, M. F. M., Silva, A. R. V. \& Damasceno M. M. C. (2011). Sobrepeso e obesidade em docentes: um estudo de prevalência. Revista de Enfermagem UFPE on line, 5(2), 193-198.

Silva, E. C, Martins, M. S. A. S, Guimarães, L. V., Segri, N. J., Lopes, M. A. L \& Espinosa, M. M. (2016). Prevalência de hipertensão arterial sistêmica e fatores associados em homens e mulheres residentes em municípios da Amazônia Legal. Revista Brasileira de Epidemiologia, 19(1), 38-51. http://dx.doi.org/10.1590/1980-5497201600010004.

Silva, G. V. (2017). Variabilidade da pressão arterial: mais uma peça do quebra-cabeça. Brazilian Journal of Nephrology, 39(2), 102-103. https://doi.org/10.5935/0101-2800.20170025.

Silva, J. L. L., Andrade, L. A. F., Pereira, L. C. L. \& Silva, P. R. B. (2010). Estresse e fatores de risco para a hipertensão arterial entre docentes de uma escola estadual de Niterói, RJ. Rev Enferm UFPE on line, 4(3): 1347-1356. http://dx.doi.org/10.5205/reuol.906-8483-1-LE.0403201002.

Vasconcelos, M. O. (2011). Trabalho, o homem e a sociedade: uma reflexão filosófica a partir de Alceu Amoroso Lima. Ágora filosófica, 1(2). https://doi.org/10.25247/P1982-999X.2011.v1n2.p\%25p.

Xavier, F. A., Barboza, L. F., Monteiro, A. M. P., Santos, L. C. \& Oliveira, D. R. (2010). Fatores de risco cardiovascular entre docentes de uma universidade pública de Minas Gerais. Revista Mineira de Enfermagem, 14(4), 465-472. 